\title{
Modeling Light Scattering from Diesel Soot Particles
}

\author{
Patricia Hull \\ Tennessee State University \\ Nashville, TN 37203 \\ Ian Shepherd, and Arlon Hunt \\ Lawrence Berkeley National Laboratory \\ Berkeley, CA 94720
}

\begin{abstract}
The Mie model is widely used to analyze light scattering from particulate aerosols. The Diesel Particle Scatterometer (DPS), for example, determines the size and optical properties of diesel exhaust particles that are characterized by measuring three angle-dependent elements of the Mueller scattering matrix. These elements are then fitted using Mie calculations with a Levenburg-Marquardt optimization program. This approach has achieved good fits for most experimental data. However, in many cases, the predicted real and imaginary parts of the index of refraction were less than that for solid carbon. To understand this result and explain the experimental data, we present an assessment of the Mie model by use of a light scattering model based on the coupled dipole approximation. The results indicate that the Mie calculation can be used to determine the largest dimension of irregularly shaped particles at sizes characteristic of Diesel soot and, for particles of known refractive index,
\end{abstract}


tables can be constructed to determine the average porosity of the particles from the predicted index of refraction.

\section{Introduction}

The environmental and health issues associated with the presence of Diesel exhaust soot particles have become of increasing concern. They have been implicated in global warming, decreased atmospheric visibility and have been classified as a toxic material by the State of California. Epidemiological studies have also shown that aerosols containing particles less than 2.5 microns are most hazardous to health. ${ }^{1,2}$ These studies led the EPA, in 1998, to mandate new atmospheric rules and regulations for 'fine' particles with diameters less than 2.5 microns. ${ }^{3}$ Diesel engine emissions are one of the most important sources of such particles. ${ }^{4,5}$ and if regulations are to be enforced and industry respond to the new regulations, it is critical that particulate emissions can be characterized rapidly and precisely.

Most current methods to characterize aerosol particle sizes are not suitable for detailed measurements because they provide only total mass, require long times for measurement or analysis, modify the particle shape or degree of agglomeration, or provide sizes based on aerodynamic sizes. To meet this need we have developed a new light scattering instrument that provides size distribution and well as compositional information from the optical properties of the particles. The Diesel Particle Scatterometer (DPS) simultaneously measures the angle dependence of three polarization components of the 
light scattering from Diesel particles. ${ }^{6}$ To interpret the experimental data, the DPS uses an optimization scheme using Mie scattering calculations to obtain a fit to all three data sets simultaneously. The input parameters to this fit provide the real and imaginary indices of refraction as well as the particle size distribution. ${ }^{7}$ The use of a Mie model to interpret such experimental data has found wide application ${ }^{8}$ but care must be taken in the use of this model due to its underlying assumptions outlined below.

The purpose of this paper is to illustrate that the results of the measurements and data analysis can be interpreted physically in terms of the particle parameters. In particular, we explore the effects of particle porosity on the polarization signals by comparing results from the coupled-dipole model for partially filled spheres with those returned from the Mie calculations. In this way, we can determine the particle porosity from the data. In addition, also using the coupled dipole model, we are able to show that our method can be used to determine the approximate effective size and index of refraction of an ensemble of moderately non-spherical particles over a limited size range characteristic of Diesel particles. To understand the measurement technique and how the data are analyzed we first describe the Mueller scattering matrix, then briefly review the operation of the DPS.

\section{Theoretical Basis of the Data Analysis}

The angle dependence of the linear and circular polarization of light scattered by a medium (in this case diesel particles) depends upon the size distribution, complex refractive index, and shape of the particles making up the medium (exhaust plume). To 
analyze how light is scattered consider the incoming and scattered beam of light. The intensity and polarization state of any beam of light are described by a 4-element Stokes vector, I, the components of which measure the following:

$$
\begin{aligned}
& \mathrm{I}=\text { total intensity of light, } \\
& \mathrm{Q}= \pm 90^{\circ} \text { polarization, } \\
& \mathrm{U}= \pm 45^{\circ} \text { polarization, } \\
& \mathrm{V}=\text { circular polarization. }
\end{aligned}
$$

The scattering of light is described by a transformation of the Stokes vector by a $4 \times 4$ Mueller matrix, $\mathbf{I}^{\prime}=\mathbf{M} \mathbf{I}$, where the $\mathbf{M}$ is a 16-element Mueller scattering matrix and $\mathbf{I}^{\prime}$ is the Stokes vector of the scattered light. The angle dependence of the elements in the matrix represent all the information available in elastically scattered light (without wavelength shift) from any scattering system, see reference 9 and 10 and their bibliographies. The scattering matrix is determined by the characteristics of the medium, in this case the particle size, structure, symmetry, orientation, and complex refractive index $m(m=n+i \kappa)$, where $n$ is the real part refractive index and $\kappa$ is the imaginary (absorptive) part of the refractive index. The scattering matrix associated with a suspension of aerosol particles is used to describe and quantify the effects of the suspension on polarized light. The elements of the Mueller matrix (here normalized by the total intensity, $S_{11}$ ) are designated in bold type as $\mathbf{S}_{\mathbf{x y}}=\mathrm{S}_{\mathrm{xy}} / \mathrm{S}_{11}$. For randomly orientated aerosol particles, the eight off-diagonal block elements of the Mueller matrix are zero. Of the remaining elements $S_{11}$ is proportional to the total scattered intensity, $\mathbf{S}_{22}$ 
is shape dependent, and the others have the following relationships:, $\mathbf{S}_{12}=\mathbf{S}_{\mathbf{2 1}}, \mathbf{S}_{\mathbf{3 3}}=\mathbf{S}_{\mathbf{4 4}}$, and $\mathbf{S}_{34}=-\mathbf{S}_{43}$.

The information that can be obtained from each matrix element is characteristic of the scattering system and is the subject of a recent review. ${ }^{10}$ In general, it is not possible at this stage in the development of light scattering theory to retrieve the size, refractive index and shape information for a distribution of particles from the observed scattering by direct inversion of the Mueller matrix. It is necessary therefore to calculate the scattering from assumed ensembles of particles using a variety of models based on the scattering system and to compare the results with the experimental data ${ }^{10}$. If randomly oriented particles are spherical or deviate from sphericity by a small fraction of the wavelength of probing light, then the scattering of such an ensemble can be calculated using Mie scattering codes. ${ }^{9}$ In this case, for spherical particles, it is only necessary to calculate four elements of the scattering matrix - all others being zero, equal to, or the negative of the four calculated, and $\mathbf{S}_{22}$ is unity. The measured value of $\mathbf{S}_{22}$ may be used as a check on the assumption of spherical symmetry. If $\mathbf{S}_{22}$ is found to be less than 0.9 at 120 degrees scattering angle, the Mie analysis is not appropriate. ${ }^{11,12}$ If the particles are nearly spherical by this criterion, agreement between observation and calculations based on the Mie model is excellent. ${ }^{8}$

\section{Description of the DPS}

The DPS is based on the same principles as the angle scanning polarization nephelometer developed earlier $^{7}$ and measures particle characteristics in diesel exhaust as a function of 
engine type, load, RPM, fuel composition, and post-combustion processes (aftertreatment, dilution, etc.). A schematic of the instrument is shown in Figure 1. It differs from the earlier instrument in that it has an array of fixed detectors instead of a single scanning detector, providing a much higher scan rate. It is capable of making the measurements in situ and evaluating the results within a few seconds. A sample of diesel exhaust is diluted and passed through the central axis sample region of the DPS. The light source for these experiments is a doubled Nd-YAG laser operating at $532 \mathrm{~nm}$. Before the light interacts with the particle stream, it passes through a polarizer and an acousto-optic modulator (Hinds Model PEM-90) that modulates the polarization (not the intensity) of the beam at a $50 \mathrm{kHz}$ rate from linear to a retardance of \pm 139.7 degrees. This modulated beam then scatters from the particles in such a way as to encode the resulting signal with three frequency components, DC, $50 \mathrm{kHz}, 100 \mathrm{kHz} .^{7}$ These three components are direct measurements of the three Mueller scattering matrix elements $S_{11}$, $\mathrm{S}_{12}$, and $\mathrm{S}_{34}$, (see above) as functions of the scattering angle.

The field of view of the detectors is defined by a series of holes in a cylindrical baffle centered about the flowing aerosol stream. The photomultipliers are arrayed concentrically around the axis of the cylinder, and measure the scattered light at 13 angles as shown in Figure 1. All but one are provided with polarizers orientated at 45 degrees to the scattering plane. The detector at the scattering angle of 120 degrees is equipped with a polarizer that is orientated parallel to the scattering plane to provide a single angle measurement of $\mathbf{S}_{22}$. A multiplexer directs the detector outputs sequentially to an $\mathrm{A} / \mathrm{D}$ converter. The three frequency components are analyzed by the copyrighted 
software developed for the instrument. The measurement of the three elements and corresponding computer data analysis require only one scan through the angles with the multiplexer. The rapid response time of the DPS (greater than $1 \mathrm{~Hz}$ data acquisition rate) allows for the measurement of particle characteristics during engine transients.

\section{Data analysis in the DPS}

In this section the application of the Mie model to the experimental output data is briefly outlined.

The output from the photomultipliers is digitized and the three matrix elements obtained by digital phase-lock amplification are computed and plotted as a function of angle. Our experimental evidence from modern, direct injection diesel engines indicates that the particles display spherical symmetry (as determined by the measured value of $\mathbf{S}_{\mathbf{2 2}}$ ) and therefore justifies the use of Mie calculations for the scattering model. The experimental values of $S_{11}, S_{12}$, and $S_{34}$ as functions of scattering angle are then fitted simultaneously by changing one set of input parameters to the Mie calculations. This procedure is carried out using the Levenburg-Marquardt (L-M) optimization technique. ${ }^{13}$ The input parameters for the code are the size distribution (mean and standard deviation for lognormal), minimum, and maximum particle diameters and the complex refractive index (m $=\mathrm{n}+i \kappa)$. The algorithm iterates to the optimal solution based on the simultaneous minimization of $\chi^{2}$, the "goodness of fit" parameter for the combined angle dependence of all three measured matrix elements. In the calculations, the minimum and maximum particle radii were fixed and other parameters allowed to vary. Results from other diesel exhaust particle measurements indicated that the distribution is best represented as 
lognormal. The best fit yields the mean and standard deviation of the size distribution and the refractive and absorptive optical properties of the particles. The DPS thus supplies information on the particle size, composition and loading, (the loading is determined from the total scattered intensity). An additional check on the veracity of the analysis arises naturally from the character of the Mie solutions. Because the simultaneous fitting of three different matrix elements is required, it is extremely unlikely that a reasonable fit to the data can be achieved from substantially non-spherical particles.

\section{Non-spherical Particles}

The underlying assumption in the Mie analysis of the data from the DPS is that the aerosol particles are spherical. The shape of the soot particles in the exhaust stream is uncertain. However, electron micrographs of soot particles collected on filters ${ }^{14}$ show that these particles are usually agglomerates of many, sometimes hundreds, of smaller, very nearly spherical carbon particles (spherules) on the order of 20 to $40 \mathrm{~nm}$ in diameter. The question arises whether is it reasonable to rely on a fitting program based on Mie calculations if the soot particles in the aerosol are irregularly shaped agglomerates. An indication that our approach is valid can be obtained from theoretical predictions of light scattering from collections of randomly oriented non-spherical particles. The resulting scattering, averaged over many spatial orientations, although not identical with the light scattered from spheres has many similar properties. We modeled the scattering from an irregularly shaped particle using the coupled-dipole approximation. (See next section for the coupled dipole model used in the calculations.) We built an ellipsoid of dipoles with a major axis about three times as long as its minor axis, and then randomly removed about 
$40 \%$ of the dipoles, creating an irregularly-shaped particle. The Mueller matrix elements were then calculated as functions of scattering angle for the particle at a single orientation and for the matrix elements averaged over the Euler angles. It can be shown that the scattering from a single particle averaged over many different spatial orientations is equivalent to the scattering from a collection of identical particles. The result of the calculations is shown in Figure 2. Also shown are the matrix elements for scattering from a sphere from a Mie calculation. The scattering from the orientation average shows the same symmetry as the Mie calculation; both $\mathbf{S}_{12}$ and $\mathbf{S}_{34}$ are zero at scattering angle of $0^{\circ}$ and $180^{\circ}$. It would be impossible to match the scattering from a sphere to the single particle due to this lack of symmetry.

\section{A. Coupled-dipole Model}

If we assume that each spherule is a dipole oscillator, the light scattering from the agglomerated particle can be calculated using the coupled-dipole approximation. ${ }^{15}$ In this model, discrete elements are placed on a cubic lattice that approximates the shape of the particle of interest. Each element on the lattice (a spherule) is treated as a spherical, dipolar oscillator with a scalar polarizability. Interactions between dipole elements are included by summing the field at a particular dipole due to the incident field and the fields induced by all the other dipoles. Calculating the interaction between dipoles leads to a large set of simultaneous linear equations (usually in matrix form) that must be solved for the electric field due to each oscillator. The scattered field is the sum of the fields arising from each oscillator. The Mueller matrix is calculated from the components of this scattered electric field using the definitions of the Stokes vectors as functions of 
the scattered field components. The light scattered from a collection of particles is calculated by performing an orientation average over Euler angles of the scattering from a single particle. ${ }^{16}$ Singham and Salzman ${ }^{17}$ found that the accuracy of the coupleddipole model depends strongly on the size of the dipole element with respect to the wavelength of the incident light. Calculations using the coupled-dipole model began to deviate from Mie calculations significantly when the individual dipoles were larger than one-tenth the wavelength of light. The size of the spherules (20 to $40 \mathrm{~nm}$ in diameter) place them within the limits of the accuracy of the method when the wavelength of the light being scattered is on the order of $500 \mathrm{~nm}$ (visible light.) In this study, $40 \mathrm{~nm}$ dipoles were used; further reducing the size of the dipoles did not have a significant affect on the calculated scattering patterns. A particle of any size can be modeled in theory, although when a large number of dipoles are needed to describe a particle accurately, the computational time and memory requirements of the computer may become prohibitively large. The electron micrographic evidence indicates that the soot particles probably contain fewer that 200 of the individual small carbon spheres, a number well within the computational limits of a modern desktop computer.

\section{B. Porous Spheres and Ellipsoids}

To validate the Mie model we used to fit the experimental data, we first used the coupleddipole approximation with orientation averaging to calculate the scattering from a sphere simulated by an array of 257 coupled- dipoles. We selected a sphere well within the accuracy of the model with size parameter 1.76 , real index of refraction 1.67, and imaginary index of refraction (absorption) of 0.7; values consistent with experimental measurements of 
solid carbon. The calculated matrix elements as functions of scattering angle were then used as simulated data and input to the DPS-based computer L-M fitting routine. The fitting routine used the Mie calculation to determine the mean radius, standard deviation, real and imaginary indices of refraction that best fit the input functions. Figure 3 shows this 'best fit' Mie calculation plotted on the same graph as the coupled-dipole calculation. The resulting output parameters were very close to our input parameters for the CD calculation, thus validating the model.

To investigate how well Mie calculations can be used to analyze scattering data from small soot-like particles (porous clusters of particles with non-spherical symmetry) we carried out numerical experiments using coupled dipole calculations. Porous particles with spherical or ellipsoidal shapes (obtained by rotating an ellipse about the major axis) were modeled by removing dipoles at random from fully dense spheres or ellipsoids. Dipoles were selected for removal from the lattice using a pseudo-random number generator that produced the same sequence of random numbers for the same initial seed. The generator allowed us to change the locations of the vacancies in the lattice or reproduce a given lattice. The matrix elements were calculated and supplied to the fitting routine to determine the best-fit parameters as before. We began from the $100 \%$ filled sphere, removing about a tenth of the dipoles and calculated the scattering elements for use in the fitting routine. We continued this process, each time removing an additional tenth of the original until we reached a structure having about $10 \%$ of the original number of dipoles and very little resemblance to a sphere. Figure 4 illustrates how the shape of a spherical cluster changed as dipoles were removed. The number of lattice points in the figure was chosen for illustration purposes 
only. The number of dipoles used in the scattering calculations, even for the smaller densities, generally exceeded 200. The CD-calculations of four scattering matrix elements, $\mathrm{S}_{11}, \mathrm{~S}_{12}, \mathrm{~S}_{22}$, and $\mathrm{S}_{34}$ as the density of the sphere is decreased are shown in Figure 5. Note that the deviation from 1.0 of $S_{22}$ increases as the sphere becomes more porous and less spherical, but is above 0.95 at all angles.

The calculated sets of matrix elements for a given porosity was then supplied to the L-M fitting routine. The L-M fit routine returned an interesting result. The diameter of the sphere predicted by the fit did not change appreciably as the sphere became more porous. Generally, the diameter predicted by the best fit tended to be the greatest distance across the lattice occupied by dipoles. When the density of dipoles became so small that few dipoles occupied lattice points far from the center of the lattice, the Mie calculation generally was able to fit the scattering data with a smaller diameter. The index of refraction and the absorption returned by the L-M routine for each sphere, however, decreased as the sphere became more porous. The mean radii, and relative real and imaginary indices of refraction, are plotted as a function of the percentage of dipoles remaining in the lattice of the sphere in Figure 6. A least squares fit to the data for index of refraction and absorption indicate a linear relationship to a high degree of confidence.

We next modeled the scattering from ellipsoids with ratios of major to minor axis (aspect ratio) of 1.5, 2.0, 3.0 and 4.0 using the same procedure we used with spheres. The size parameter was kept constant at 2.0 for all ellipsoids. The size parameter of an ellipsoid is defined in the same way as for a sphere, $x=2 \pi r / \lambda$. In this case, $r$ is the effective radius of 
the ellipsoid, that is, the radius of a sphere having the same volume. The CD-calculations of four scattering matrix elements, $\mathbf{S}_{11}, \mathbf{S}_{12}, \mathbf{S}_{22}$, and $\mathbf{S}_{34}$ for each porosity of the ellipsoid are shown in Figure 7. The graphs bear a strong resemblance to the graphs for scattering from spheres except for $\mathbf{S}_{22}$. The values were generally lower, indicating a larger deviation from spherical, but it still did not drop blow 0.90. Unlike the sphere, however, $\mathbf{S}_{22}$ increased as the ellipsoid became more porous. Thus the more porous the ellipsoid became, the more spherelike its scattering.

Each set of matrix elements as a function of scattering angle for an ellipsoid of a given porosity was supplied to the L-M fitting routine. The mean radius, and the real and imaginary refractive index predicted by the L-M routine are plotted as a function of the percentage of dipoles remaining in the lattice of the ellipsoid (fill density) are shown in Figure 8. As with the sphere, the radius remained nearly constant except for the first two points when the lower density reduced the outer perimeter of the particle. The relative index of refraction and absorption were linear functions of the fill density with a high confidence level. The radius predicted by the routine was slightly smaller than the effective radius of the ellipsoid but nearly constant at all densities.

\section{Discussion and Results}

We have established a simple relationship between the parameters returned by the fitting routine of the DPS and the particle properties. Since the particles we are modeling consist primarily of one material, carbon, we know the values of the complex index of refraction. Therefore, the graphs in Figures 6 and 8 can be used with the real and 
imaginary indices of refraction calculated from the L-M routine to determine the approximate porosity of the particles in the scattering medium.

If we use $\mathbf{S}_{22}$ above 0.9 at all scattering angles as the criterion for a good Mie fit, we can establish some limits based on CD calculations for the size and shape of particles we can model accurately by the Mie fitting program. The three graphs in Figure 9 illustrate the approach. Note that in graph (a) for filled lattices, ellipsoids with a size parameter of 2.0 and aspect ratios of 2.5 and higher would not be well modeled with Mie calculations. Other factors are important, however, such as fill density of the lattice as graph (b) shows. Note that even for a size parameter of 2.0 and an aspect ratio of 4.0, we should be able to fit Mie calculations to the scattering for lattices that are 50\% or less filled. Graph (c) illustrates the effects of size parameter on $\mathbf{S}_{\mathbf{2 2}}$ for ellipsoids with a fully filled lattice and aspect ratio of 4.0. Note that all size parameters down to a size parameter of 0.5 produce an $\mathbf{S}_{22}$ that drops below 0.9. Size parameters from 2.5 to 0.5 translate to particle diameters from about 400 to $80 \mathrm{~nm}$ for scattering of green light (532nm). Even for small particles, if they were elongated, we would not expect to get a good fit to the scattering pattern with a Mie calculation unless they are porous. $\mathbf{S}_{\mathbf{2 2}}$ for diesel particles measured with the DPS were on the order of 0.95 indicating that the soot particles are probably not very elongated. The low indices of refraction and absorption returned by the L-M routine for experimental data also indicate that the particles are small and porous. We were able to get good fits for the ellipsoids with aspect ratios of 1.5 and 2.0. The fits at most densities for the aspect ratio of 3.0 were not quite as good but still acceptable. For an aspect ratio of 4.0, the LM routine produced poor fits or no reasonable fits at all. 


\section{Conclusion}

Numerical experiments were carried out using coupled dipole calculations with orientation averaging to investigate how well Mie calculations could be used to analyze scattering data from small soot-like particles. Porous spherical or ellipsoidal particles were modeled by removing dipoles at random from fully dense spheres or ellipsoids. We were able to model spheres, and ellipsoids with aspect ratios up to 3.0, with any fill density. As the fill density of the particle decreased, the predicted values of the index of refraction and absorption from Mie calculations decreased linearly. The predicted radius of the particle remained nearly constant for spheres and for ellipsoids up to aspect ratios of 3.0. At an aspect ratio of 3.0, the Mie fit predicted slightly reduced radius as the porosity decreased.

$\mathbf{S}_{22}$ proved to be a good indicator of how well the particle could be represented by a Mie calculation. If the value of $\mathbf{S}_{\mathbf{2 2}}$ for a particle dropped below 0.9 at any angle, Mie fits were generally poor or impossible to obtain. If a particle sample met the criterion, a determination of the mean diameter, index of refraction, and absorption from the L-M routine for the sample, coupled with graphs similar to figures 7 and 9 gave a good indication of the particle’s longest dimension and average porosity.

Due to the extensive use of Mie approximations in the analysis of light scattering from aerosols of many types these results are significant for the interpretation of such data 
where, as is often the case, the optical properties and the morphology of the particles are not well characterized.

\section{Acknowledgements}

This work was supported by the Assistant Secretary for Energy Efficiency and

Renewable Energy, Advanced Automotive Office of Transportation Technologies of the United States Department of Energy under Contract No. DE-AC03-76SF00098. 


\section{References}

1. C.A. Pope, III, D. Dockery and J. Schwartz, "Review of Epidemiological Evidence of Health Effects of Particulate Air Pollution," Inhalation Toxicology, 7, 4-18 (1995).

2. D.S. Shprentz, , "Breath-taking: Premature mortality due to particulate air pollution in 239 American cities," NRDC Publications Department, 40 West 20 Street, New York, NY 10011, 1996.

3. EPA, “National Air Quality and Emissions Trends Report,” 1994. EPA-454/R-95-011, Springfield, VA, 1995.

4. W.H. Lipkea, J.H. Johnson, and C.T. Vuk, "The physical and chemical character of diesel particulate emissions - Measurement techniques and fundamental considerations," Paper No. 780108, SAE International, Warrendale, PA (1979).

5. J.B. Heywood, Internal Combustion Engine Fundamentals, New York, McGraw-Hill, 1988.

6. A.J. Hunt, M.S. Quinby-Hunt, I.G. Shepherd, "Polarized Light Scattering for Diesel Exhaust Particulate Characterization," Proc. of the Diesel Engine Emissions Reduction Workshop, J. Fairbanks, Ed., DOE/EE-0191, 1999 
7. A. J. Hunt and D. R. Huffman, “A New polarization-modulated light scattering instrument.” Rev. Sci. Instrum. 44, 1753-1762 (1973).

8. C.F. Bohren, and D.R. Huffman, Absorption and Scattering of Light by Small Particles, Wiley, New York (1983).

9. R.J. Perry, A.J. Hunt, and D.R. Huffman, "Experimental Determination of Mueller Scattering Matrices for Nonspherical Particles," Appl. Opt., 17, 2700-2710 (1978).

10. M.S. Quinby-Hunt, P.G. Hull, and A.J. Hunt, "Polarized Light Scattering in the Marine Environment," Chapter 19, Light Scattering by Non-Spherical Particles: Theory, Measurements, and Geophysical Applications, M.L. Mischenko, J.W. Hovenier, and L.D. Travis, Eds. Academic Press (2000).

11. M.S. Quinby-Hunt, P.G. Hull, and A.J. Hunt, "Predicting Polarization Properties of Marine Aerosols," Ocean Optics XII, Proc. SPIE, 2258, 735-746 (1994).

12. M.S. Quinby-Hunt, L.L. Erskine, and A.J. Hunt, "Polarized Light Scattering by Aerosols in the Marine Atmospheric Boundary Layer," Applied Optics, 36, 5168-5184 (1997). 
13. W.H. Press, B.R. Flannery, S.A Teukolsky, and W.T. Vetterling, Numerical Recipes (Fortran version), 523 Cambridge University Press, Cambridge, UK (1989).

14. D.C. Sigla and G.W. Smith, Particulate Carbon, Plenum Press, New York, (1981).

15. E. M. Purcell and C.R. Pennypacker, "Scattering and absorption of light by nonspherical dielectric grains,” Astrophys. J. 186, $705-714$ (1973).

16. P. G. Hull, M.S. Quinby-Hunt, D>B $>$ Shapiro, “Underwater Imaging, Photography, and Visibility” Proc SPIE. 1537, 21-30, 1991.

17. S. B. Singham and G. C. Salzman, "Evaluation of the scattering matrix of an arbitrary particle using the coupled-dipole approximation,” J. Chem. Phys. 84, 2658-2667 (1986). 


\section{Figure Captions}

Figure 1. A schematic of the DPS. The base plate is about 2 feet square.

Figure 2. Mueller matrix elements for scattering from a single particle and an orientation average of the same particle. A Mie calculation (solid line) is also shown for comparison.

Figure 3. A comparison of three matrix elements for a sphere with a size parameter of 1.76 using the coupled-dipole calculation and a Mie calculation. The Mie calculation is the solid line without markers and the coupled-dipole calculation is marked by open circles.

Figure 4. Sketch of the arrangement of the dipoles on a lattice of 81 positions as dipoles are removed by random selection. The circular line represents the radius of a sphere used in the Mie calculation that best fits the scattering matrix elements.

Figure 5. Results of a series of coupled-dipole calculations of the scattering matrix elements for sphere with a size parameter of 1.76. Dipoles were removed from the lattice by random selection and the matrix elements recalculated. A density of $100 \%$ is a sphere with a completely filled lattice.

Figure 6. Index of refraction and absorption of a sphere. The figure shows the decrease in the index of refraction and absorption determined by the L-M fit to a Mie sphere as the CD sphere becomes more porous. The 'effective' radius determined by the LM fit is 
approximately that of $\mathrm{CD}$ sphere and the radius predicted by the model does not change appreciably is the sphere becomes more porous.

Figure 7. Results of a series of coupled-dipole calculations of the scattering matrix elements for an ellipsoid with a ratio of major to minor axis of 2.0. The size parameter of a sphere of equivalent volume is 1.89. Dipoles are removed from the lattice by random selection and the matrix elements recalculated. A density of $100 \%$ is an ellipsoid with a completely filled lattice.

Figure 8. Index of refraction and absorption of an ellipsoid with a ratio of the major to minor axis of 2.0. The figure shows the decrease in the index of refraction and absorption determined by the LM fit to a sphere as the ellipsoid becomes more porous. The 'effective' radius determined by the LM fit is approximately that of sphere made of the same number of dipoles. The radius predicted by the model does not change appreciable is the ellipsoid becomes more porous.

Figure 9. (a) Matrix element, $\mathbf{S}_{\mathbf{2 2}}$, for ellipsoids of various aspect ratios. The size parameter has been fixed at 2.0 and all ellipsoids have a $100 \%$ filled lattice. (b) $\mathbf{S}_{22}$ for ellipsoids of different fill densities. The size parameter has been fixed at 2.0 and the aspect ratio at 4.0. (c) $\mathbf{S}_{22}$ for ellipsoids with varying size parameters. All ellipsoids have a $100 \%$ filled lattice and aspect ratio is fixed at 4.0 . 


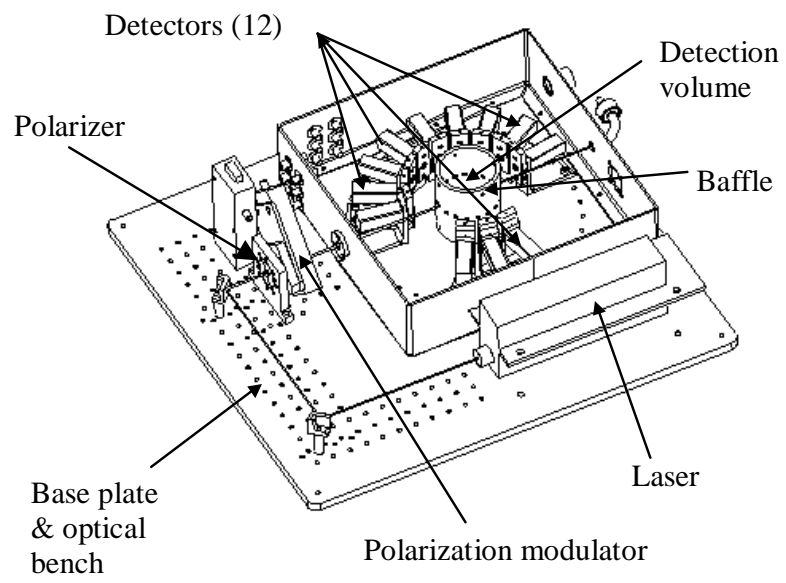

Figure 1. 

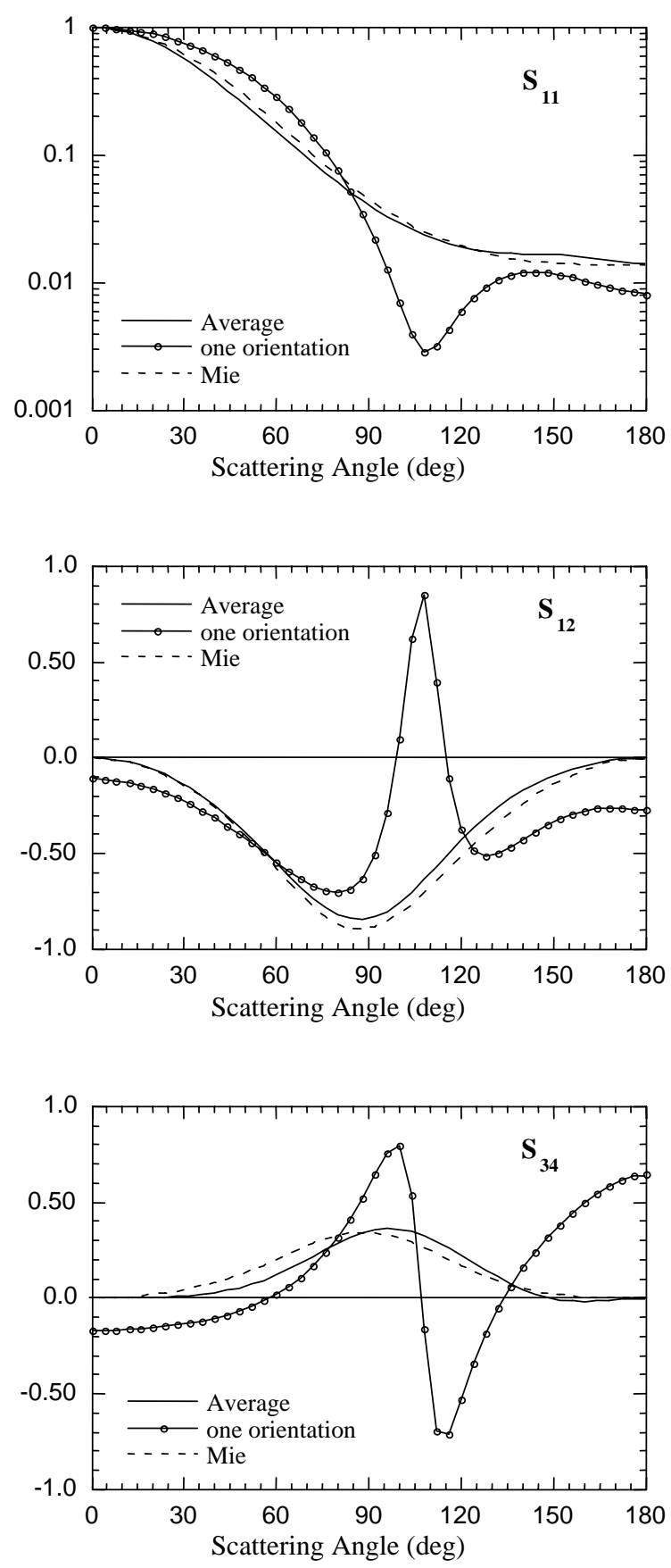

Figure 2. 

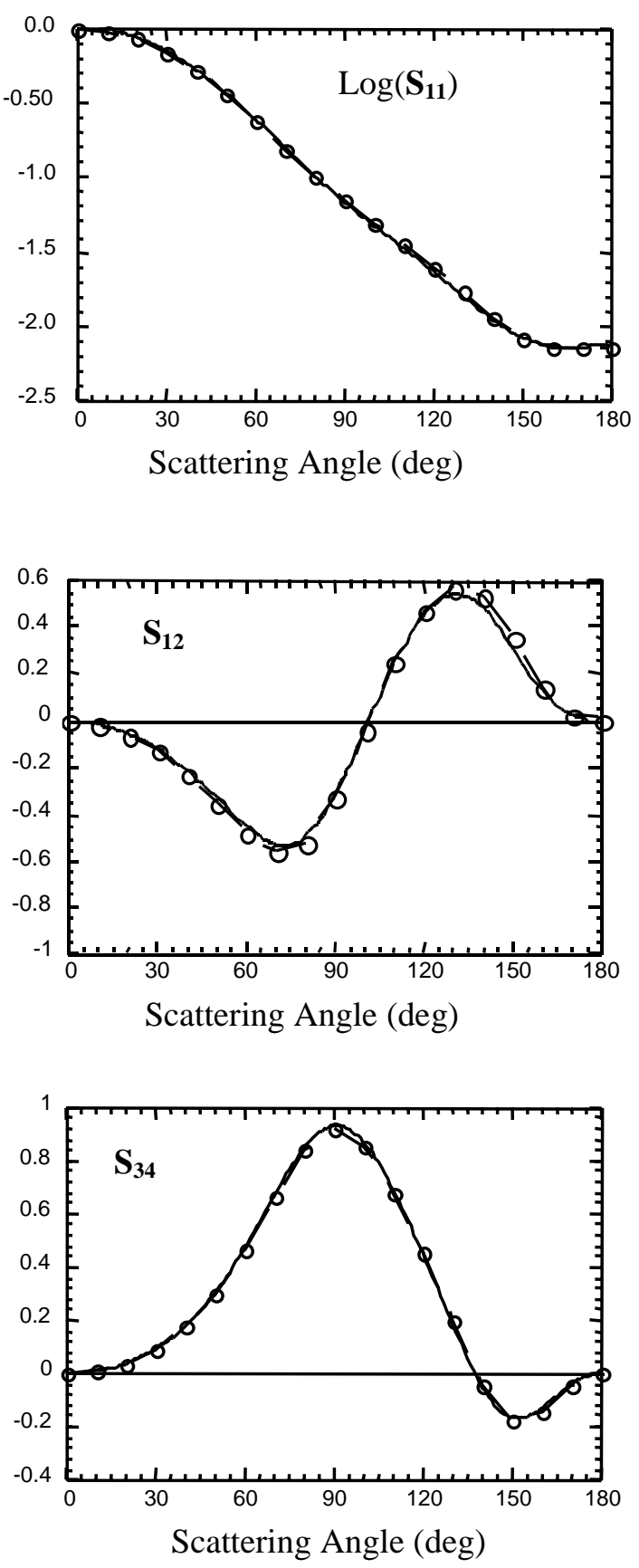

Figure 3 


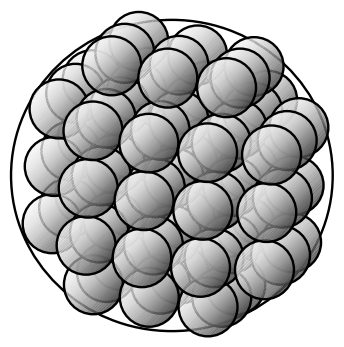

100\% Filled

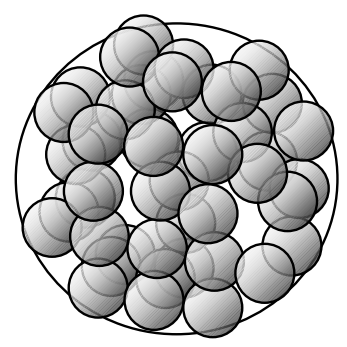

$60 \%$ Filled

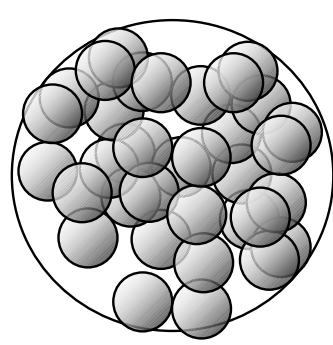

40\% Filled

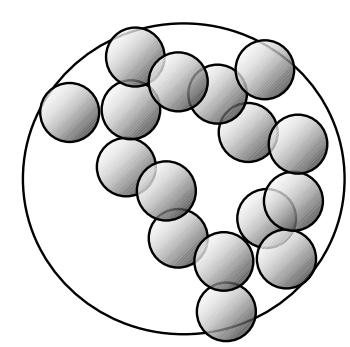

20\% Filled

Figure 4 . 

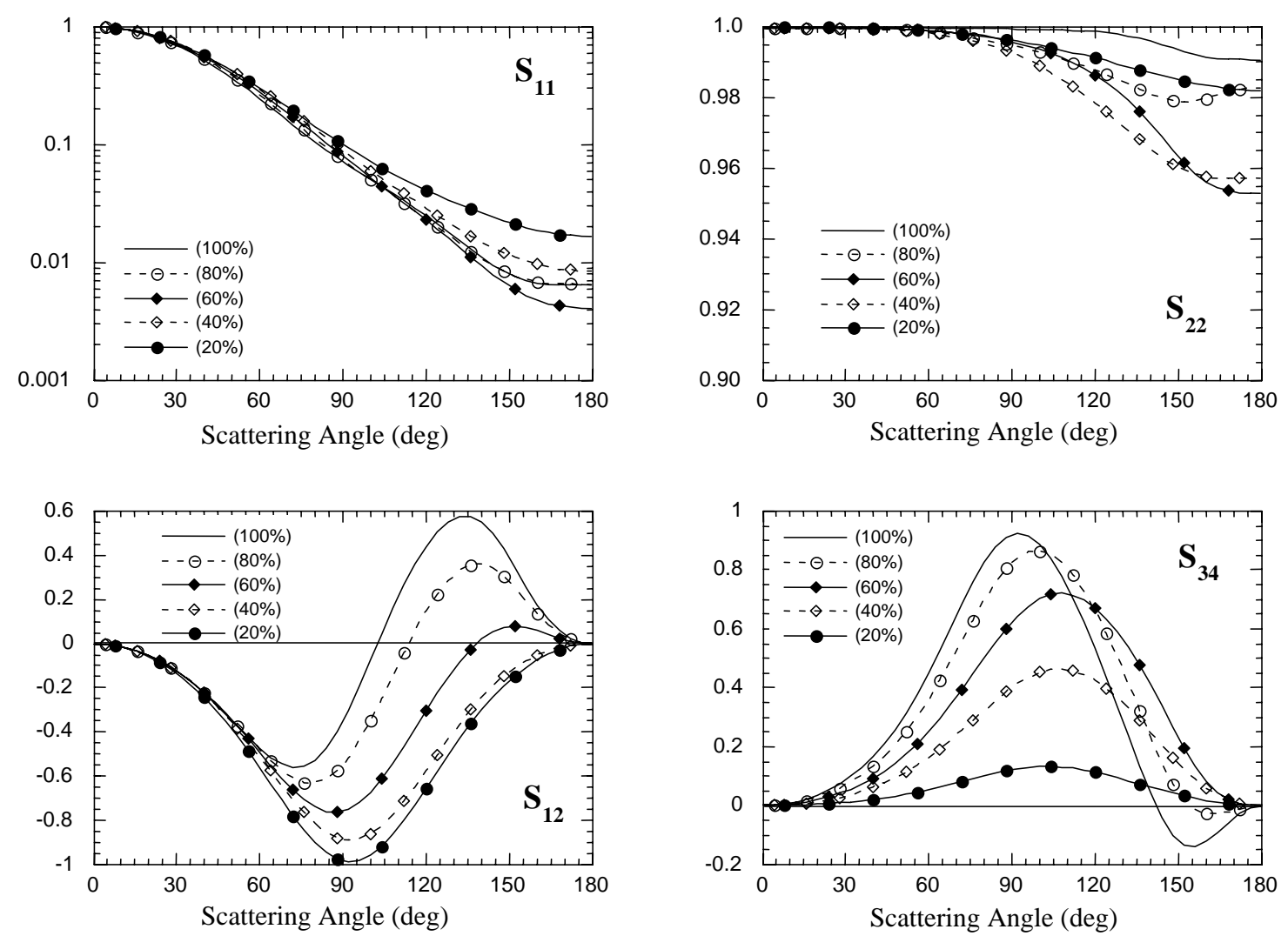

Figure 5. 

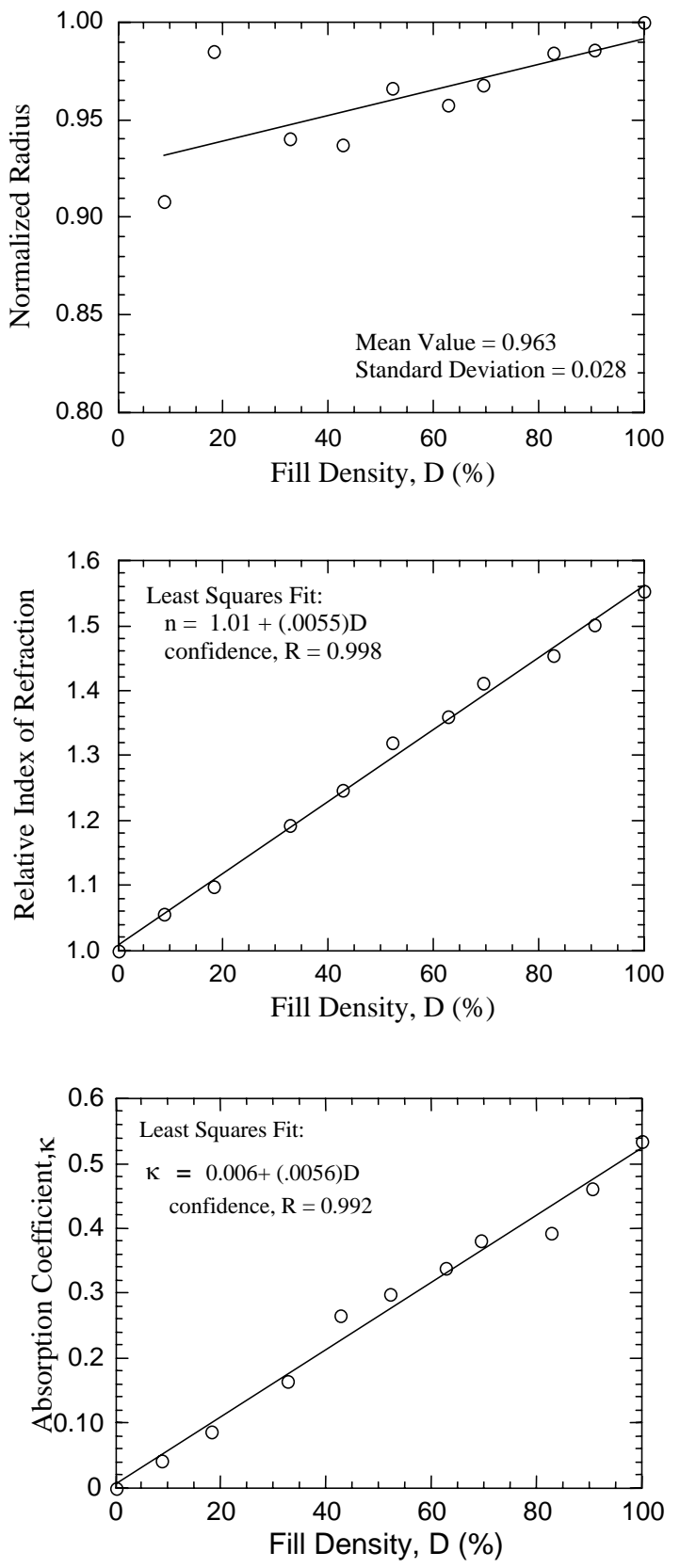

Figure 6. 

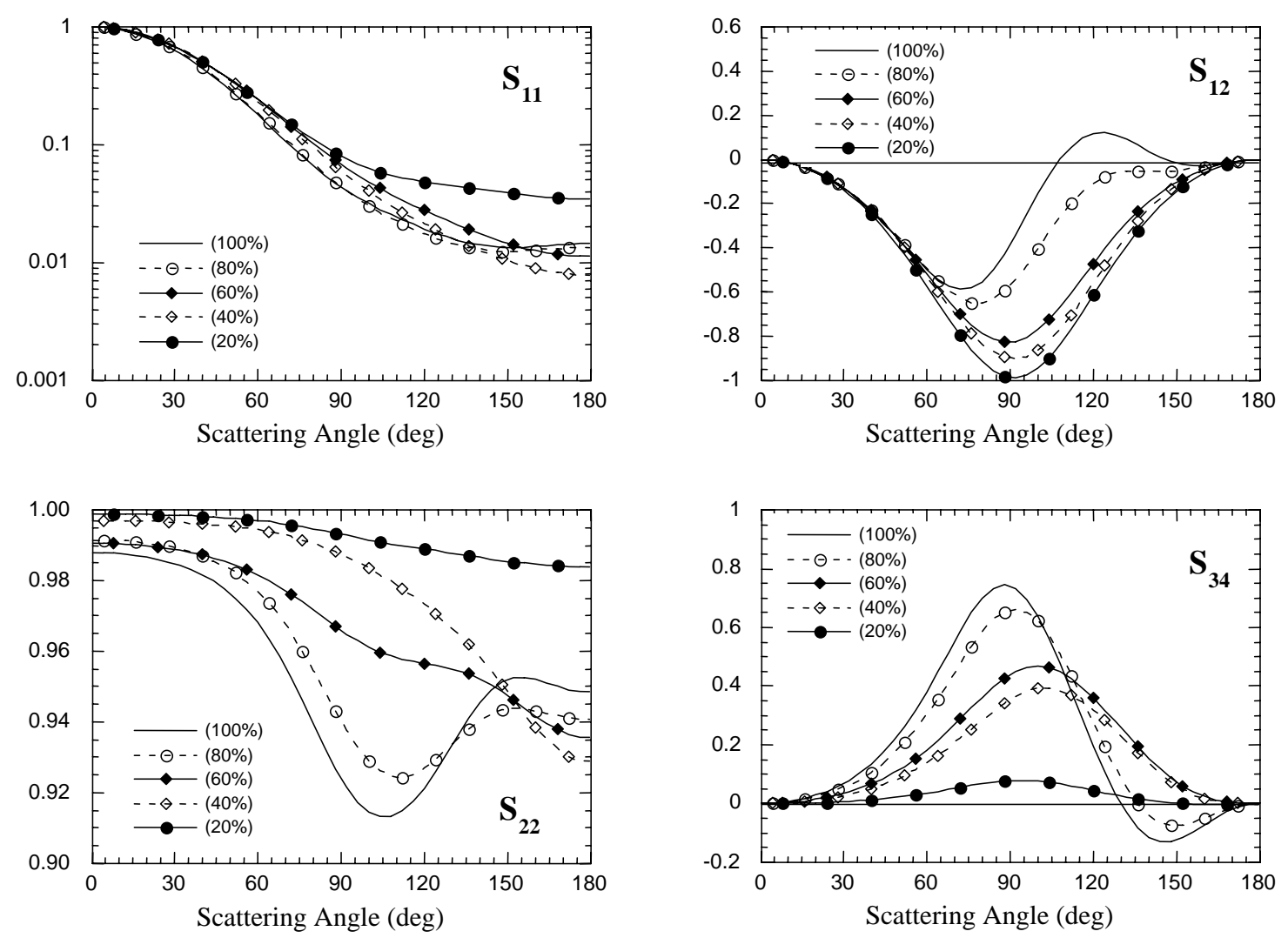

Figure 7. 

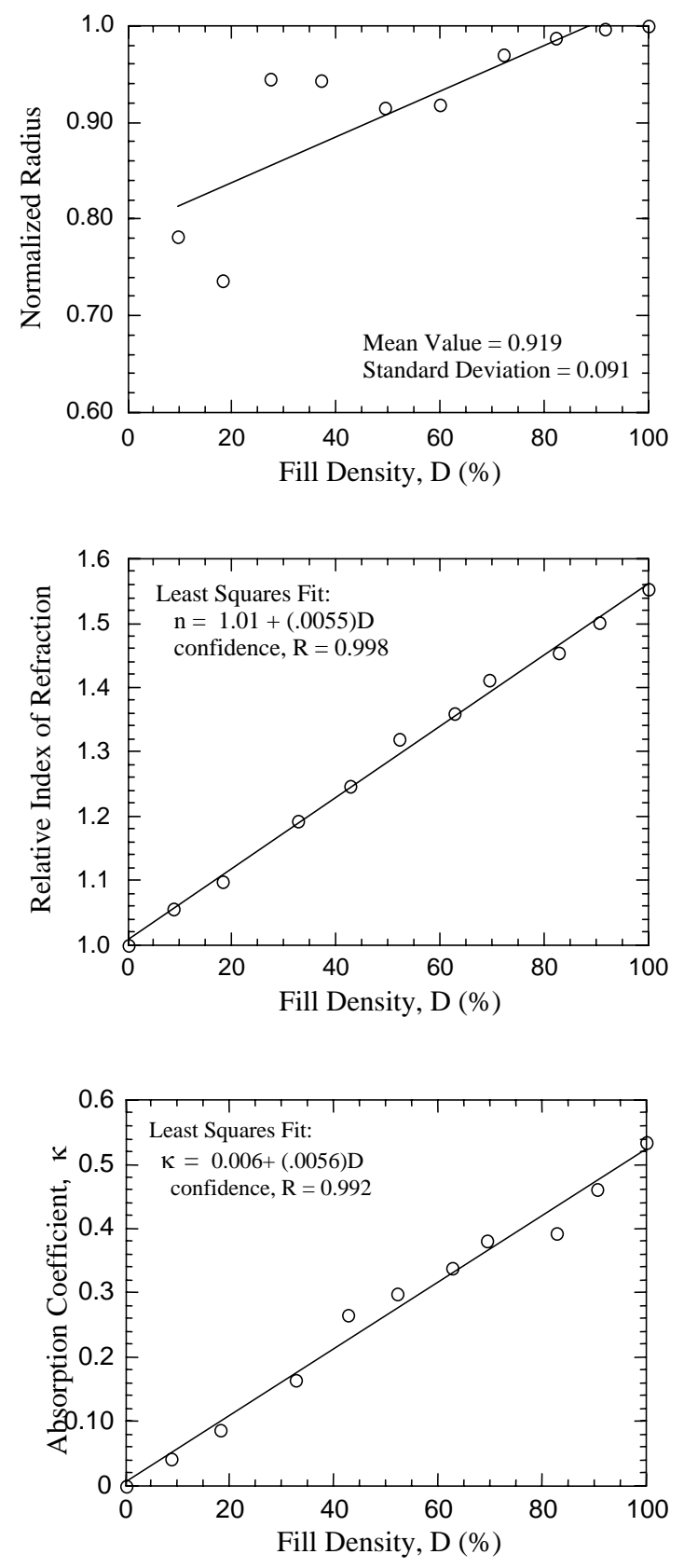

Figure 8. 

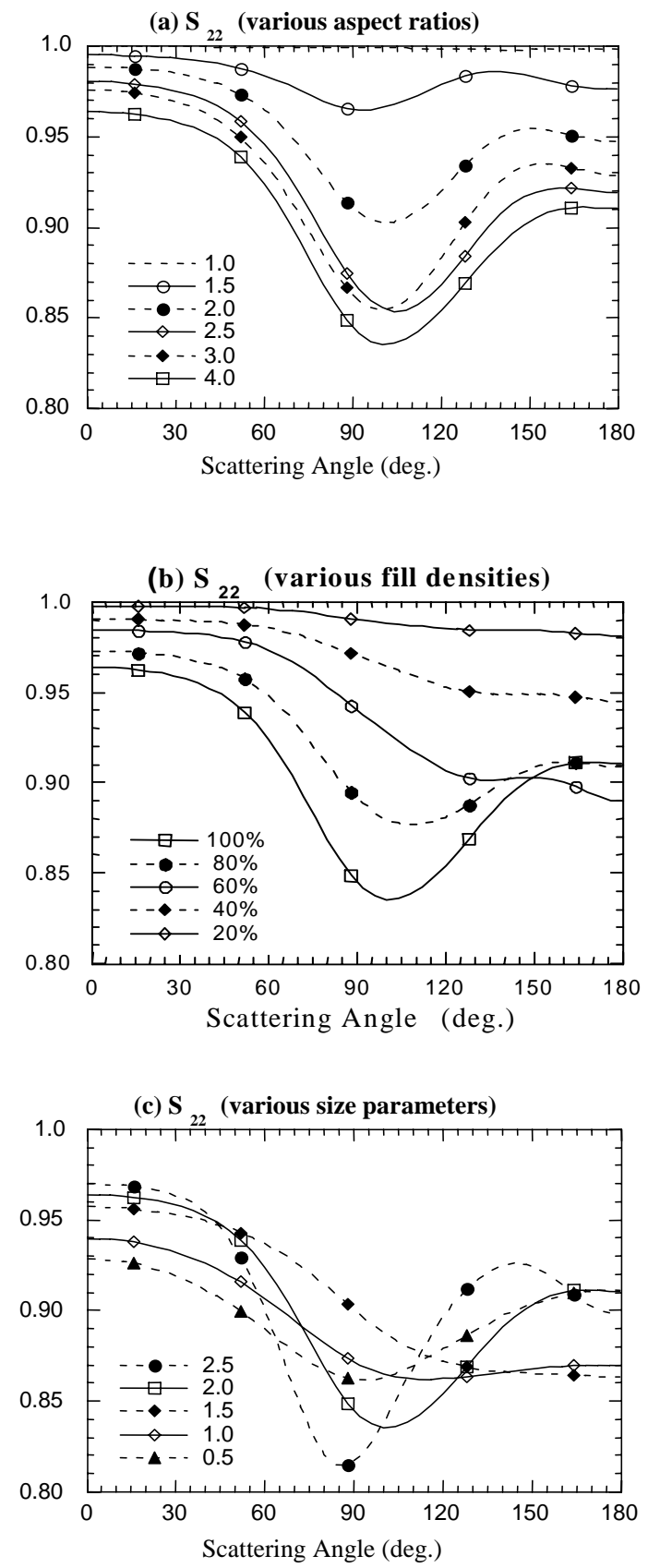

Figure 9

Figure 9. (a) Matrix element, $\mathbf{S}_{\mathbf{2 2}}$, for ellipsoids of various aspect ratios. The size parameter has been fixed at 2.0 and all ellipsoids have a $100 \%$ filled lattice. (b) $\mathbf{S}_{22}$ for ellipsoids of different fill densities. The size parameter has been fixed at 2.0 and the aspect ratio at 4.0. (c) $\mathbf{S}_{22}$ for ellipsoids with varying size parameters. All ellipsoids have a $100 \%$ filled lattice and aspect ratio is fixed at 4.0 . 